\title{
DYNAMIC COUPLING OF CONVECTIVE FLOWS AND MAGNETIC FIELD DURING FLUX EMERGENCE
}

\author{
FAng FAnG ${ }^{1}$, Ward Manchester IV ${ }^{1}$, William P. AbBetT ${ }^{2}$, And Bart VAn DER Holst ${ }^{1}$ \\ ${ }^{1}$ Department of Atmospheric, Oceanic and Space Sciences, University of Michigan, Ann Arbor, MI 48109, USA \\ ${ }^{2}$ Space Sciences Laboratory, University of California, Berkeley, CA 94720, USA \\ Received 2011 August 3; accepted 2011 October 20; published 2011 December 28
}

\begin{abstract}
We simulate the buoyant rise of a magnetic flux rope from the solar convection zone into the corona to better understand the energetic coupling of the solar interior to the corona. The magnetohydrodynamic model addresses the physics of radiative cooling, coronal heating, and ionization, which allow us to produce a more realistic model of the solar atmosphere. The simulation illustrates the process by which magnetic flux emerges at the photosphere and coalesces to form two large concentrations of opposite polarities. We find that the large-scale convective motion in the convection zone is critical to form and maintain sunspots, while the horizontal converging flows in the nearsurface layer prevent the concentrated polarities from separating. The footpoints of the sunspots in the convection zone exhibit a coherent rotation motion, resulting in the increasing helicity of the coronal field. Here, the local configuration of the convection causes the convergence of opposite polarities of magnetic flux with a shearing flow along the polarity inversion line. During the rising of the flux rope, the magnetic energy is first injected through the photosphere by the emergence, followed by energy transport by horizontal flows, after which the energy is subducted back to the convection zone by the submerging flows.
\end{abstract}

Key words: magnetohydrodynamics (MHD) - Sun: atmosphere - Sun: interior

Online-only material: animation, color figures

\section{INTRODUCTION}

The solar magnetic field emerges at the surface over a wide range of scales, from ubiquitous ephemeral regions as small as $10^{16} \mathrm{Mx}$, to active regions as large as $10^{23} \mathrm{Mx}$, which emerge at low to midlatitudes (Hagenaar et al. 2003; Parnell et al. 2009). Observations from satellites and ground-based telescopes find that the configuration of the magnetic field plays an important role in the solar eruptive events, i.e., coronal mass ejections (CMEs) and solar flares (Canfield et al. 1999). It is thus of great importance to study the building up of the magnetic field in the solar atmosphere, as it rises from the convection zone. However, the study of the transport of magnetic flux and energy has been hampered by the invisibility of subsurface structures. With the time-distance helioseismic analysis, Kosovichev (1996) and Zhao \& Kosovichev (2003) provide a view on the horizontal and vertical flow velocities on the subsurface layers under sunspots and identify shear flows and rotation of the sunspots underneath the surface, which may build up the energy and helicity in the atmosphere.

Over the past decades, the development of numerical models has also greatly improved our understanding of the dynamics and energetics of magnetic flux emergence. Shibata et al. (1989) describe a two-dimensional magnetohydrodynamic (MHD) simulation on the emergence of a horizontal magnetic flux rope from the photosphere into the chromosphere using a two-layered atmosphere. Fan (2008) and Jouve \& Brun (2009) carry out sets of anelastic MHD simulations on the buoyant rise of the magnetic flux tube from the base of convection zone to near the top, respectively. In particular, Fan (2008) shows the rotation of the flux tube driven by the Lorentz force at the two ends while the twisted tube is bent. Manchester \& Low (2000) suggested that shearing motion driven by the Lorentz force draws the magnetic field parallel to the polarity inversion line (PIL), which was demonstrated in simulations of emerging flux ropes (Fan 2001; Magara \& Longcope 2003; Abbett \& Fisher 2003;
Manchester et al. 2004; Archontis \& Török 2008; MacTaggart \& Hood 2009). Magara \& Longcope (2003) found that during emergence, energy flux through the photosphere is first dominated by the vertical flows while horizontal flows dominate the later phase. The energy transport of shear flows naturally provides an energy source for CMEs (Manchester 2007, 2008). Simulations have also revealed that shear flows driven by the Lorentz force can produce eruptions in both magnetic arcades (Manchester 2003) and emerging flux ropes (Manchester et al. 2004; Archontis \& Török 2008; MacTaggart \& Hood 2009) providing further evidence of a mechanism for CMEs, flares, and filament eruptions.

With the availability of more computational resources, numerical models are able to take into account the thermodynamic processes in the solar interior and atmosphere, and produce a realistic convection zone overlaid by a self-consistent upper atmosphere (Vögler et al. 2005; Stein \& Nordlund 2006; Hansteen et al. 2006; Abbett 2007; Gudiksen et al. 2011). Such realistic simulations reveal a wealth of complex dynamic interactions between the magnetic field and the convective flows. Cheung et al. (2007) and Tortosa-Andreu \& Moreno-Insertis (2009) study the rise of buoyant magnetic flux tubes from the near-surface convection zone into the photosphere and chromosphere and find the fundamental role of convective flows in the emergence of the magnetic flux at the photosphere. Cheung et al. (2007) and Martínez-Sykora et al. (2008, 2009) simulate an emerging flux tube, which increases the size of the photospheric granules and alters the chromospheric structure. More discussion on the interaction between the magnetic field and the convective flows can be found in Fan (2004) and Nordlund et al. (2009).

Radiative MHD simulations on a larger scale (Rempel et al. 2009; Cheung et al. 2010) report the formation of the sunspots and active regions. Rempel et al. (2009) and Rempel (2011) find outflows in the penumbral structure driven by the Lorentz forces at the surface and by the convective flows in the deeper layers. Cheung et al. (2010) simulate the formation of a pair of sunspots 
in an active region with a magnetic semi-torus advected through the bottom of the domain and find the mass removal in the magnetic flux driven by the reconnection, and the migration of the flux due to horizontal flows. With the inclusion of turbulent convection (Fang et al. 2010), it is shown that the transport of energy into the corona becomes even more critical as downdrafts return much of the magnetic energy back below the photosphere. This simulation treats the emergence of a weak axial flux of $3.3 \times 10^{19} \mathrm{Mx}$, which is quickly shredded and dispersed to the intergranular lanes by the convection motions.

These radiative MHD simulations illustrate the importance of turbulent convective flows in the emergence of the magnetic structures. In light of these previous results, we expand upon the work of Fang et al. (2010) by simulating the emergence of a larger twisted magnetic flux rope emerging from deeper in the convection zone, and study the energetics during its rise through the turbulent plasma to reside in the hot corona. With this work, we strive to more deeply understand the energetic coupling between the convection zone and the corona provided by the magnetic field. In the following sections, we present the results of our simulation. Section 2 describes the numerical methods and the simulation steps. In Section 3, we study the results by analyzing the evolution of the magnetic and energetic flux under the impact of the convective flows. Finally, we discuss the conclusions of our simulation and its implication on further simulations.

\section{NUMERICAL METHODS}

For our simulation, the MHD equations are solved with the Block-Adaptive Tree Solar-wind Roe Upwind Scheme (BATSRUS; Powell et al. 1999). Additional energy source terms are implemented to include the thermodynamic processes in the solar atmosphere, i.e., surface cooling at the photosphere, radiative cooling of the corona, and magneticflux-related heating in the corona (Abbett 2007). We describe the radiative losses in the low-density corona as optically thin radiation and artificially extend the cooling function to treat the solar surface temperature as well. The coronal heating is approximated by Pevtsov et al. (2003), which gives an empirical relationship between the heating rate and the unsigned magnetic flux at the photosphere. Our model neglects electron thermal conduction along magnetic field lines, since the simulations here focus on the transfer of energy and magnetic field from the convection zone to the corona. The application of a tabular, non-ideal equation of state takes account of the ionization and the excitation of particles in the dense and hot solar interior (Rogers 2000). Horizontal boundary conditions are periodic, and the lower boundary condition sets the density and temperature of the same values found in the initial state of the atmosphere, while keeping the vertical momentum reflective across the boundary. The upper boundary is closed. The details of the model are described in Fang et al. (2010).

\subsection{Initial Atmosphere}

To develop the coronal model, we first extract the averaged state of the solar atmosphere from the model of Fang et al. (2010), at a depth of $2.5 \mathrm{Mm}$ below the photosphere. In the convection zone, the plasma is adiabatic as the heating source is absent and radiative cooling is negligible in the optically thick medium. The entropy is then nearly invariant throughout the deep convection zone. Here the deep convection zone is defined as deep in the simulation domain, which covers the upper $10 \%(20 \mathrm{Mm})$ depth of the solar convection zone. Under the assumption that the plasma is both isentropic and in hydrodynamic equilibrium, we solve the following equations for the structure of the atmosphere in the deep convection zone with the extracted values providing the upper boundary condition:

$$
\begin{gathered}
\nabla p=\rho \mathbf{g}, \\
\log \left(\frac{p}{\rho^{\gamma}}\right)=C,
\end{gathered}
$$

where $p, \rho$, and $\mathbf{g}$ are the plasma pressure, mass density, and gravitational acceleration, respectively. $C$ is the average entropy value in the convection zone at $z=-2.5 \mathrm{Mm} . \gamma$ is the adiabatic index, which can be obtained from the tabular equation of state using the pressure and density values, $p$ and $\rho$. For the purpose of our simulation here, the atmosphere is extended to $21 \mathrm{Mm}$ below the photosphere by solving Equations (1) and (2). The stratification of the density and pressure of the extended atmosphere in the convection zone are found to be in agreement with that of Stein et al. (2011).

This solution is then used as an initial condition for a onedimensional calculation in which we apply the surface cooling. The plasma in the simulation domain then goes through an hour of thermal relaxation to form a superadiabatic background atmosphere, which is unstable to convective motion. The next step is to perform the full three-dimensional simulation with the size of the domain set to $30 \times 30 \times 42 \mathrm{Mm}^{3}$, extending $21 \mathrm{Mm}$ below the surface and $21 \mathrm{Mm}$ above in the vertical directions, while the horizontal area of the domain is comparable to the size of a small active region. In the superadiabatically stratified atmosphere, convective motion starts immediately after a small energy perturbation and relaxes over a period of roughly one turnover time of the convection extending to a depth of $21 \mathrm{Mm}$. After the convective motion has relaxed, we impose an initial vertical magnetic field $B_{z}$ of $1 \mathrm{G}$ to the domain and turn on the heating and radiative cooling term in the coronal region. With the magnetic-flux-related coronal heating term (Pevtsov et al. 2003), the coronal temperature is heated up to $1 \times 10^{6} \mathrm{~K}$ within $2 \mathrm{hr}$.

The left panel in Figure 1 shows the averaged vertical stratification of the density and temperature in the simulation domain. The density drops by about five orders of magnitude from the bottom of the domain to the photosphere, and the temperature drops by two orders of magnitude. Above the photosphere, the density drops by another seven orders of magnitude, and the temperature increases to $1 \mathrm{MK}$ within $2 \mathrm{Mm}$. The strong variation in the atmospheric stratification puts large requirements on spatial resolution and restrictions on the explicit time step, given the high temperatures and subsequent sound speeds. However, the application of the adaptive mesh refinement in BATSRUS greatly facilitates the simulation in saving computational resources while keeping the necessary refinements to resolve the local turbulent structures. The grid size in our simulations has three levels of refinement with cubic cells of size $37.5 \mathrm{~km}, 75 \mathrm{~km}$, and $150 \mathrm{~km}$. The grid is refined in horizontal layers with higher resolution near the surface where temperature and density gradients are largest.

The right panel in Figure 1 shows the variation of the vertical velocity $u_{z}$ at $z=-3 \mathrm{Mm}$. Red lines outline the regions with concentrations of $B_{z}$ field, which coincides well with the downflowing area. The right panel in Figure 2 illustrates the structure of $u_{z}$ on a vertical cut of the convection zone. 

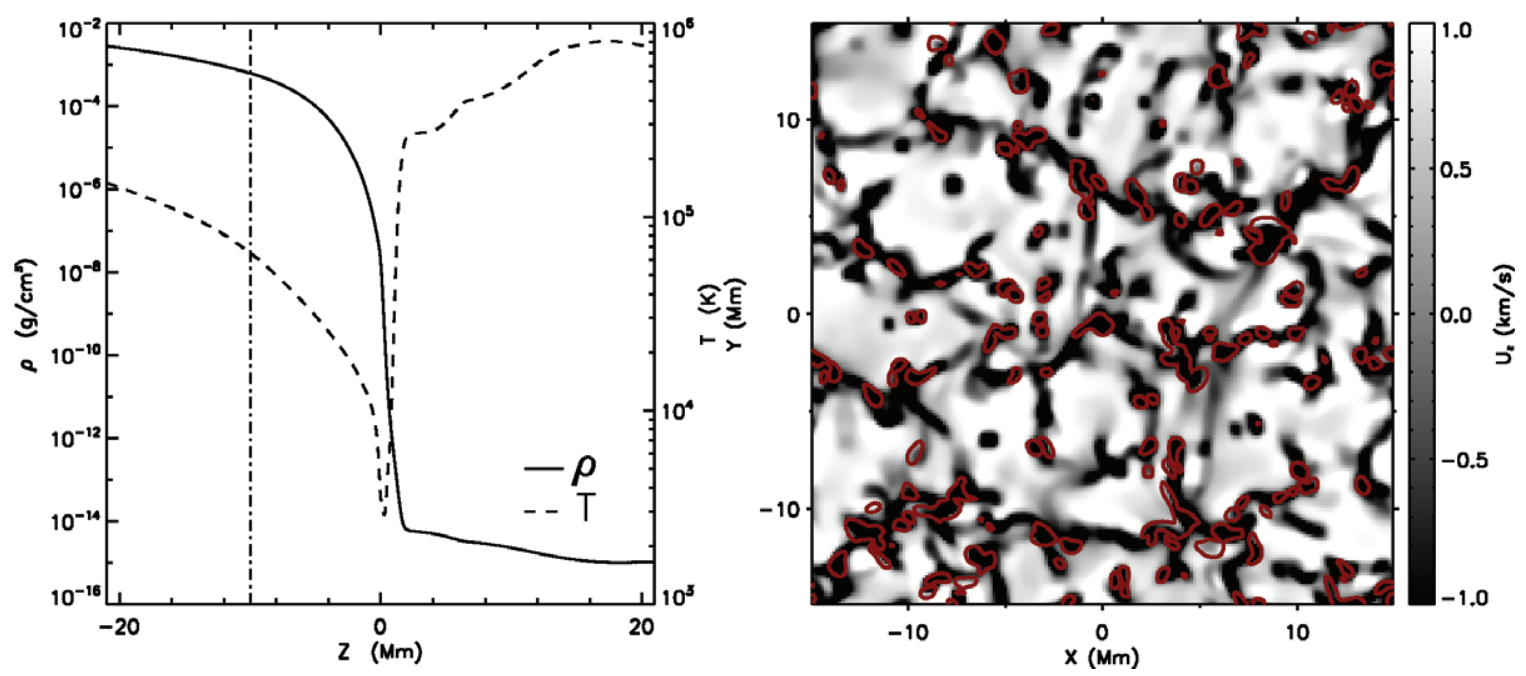

Figure 1. Left: the vertical stratification of the density (solid) and the temperature (dashed) of the solar atmosphere. The dash-dotted line indicates the initial location of the inserted flux rope. Right: the vertical velocity structure at $z=-3 \mathrm{Mm}$. Red lines show regions with $\left|B_{z}\right|$ greater than $10 \mathrm{G}$.

(A color version of this figure is available in the online journal.)

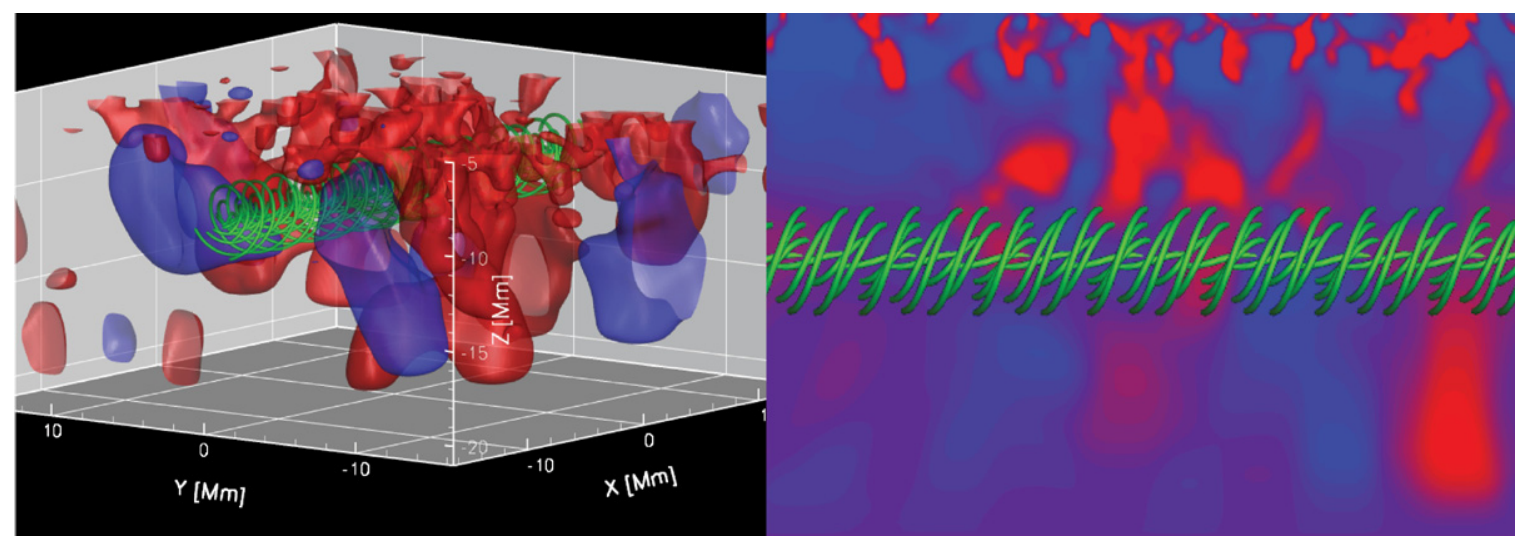

Figure 2. Initial structure of magnetic flux rope with color showing the vertical velocity in the convection zone. Left: magnetic flux rope embedded in the convective flows with blue isosurfaces indicating upflows at $1 \mathrm{~km} \mathrm{~s}^{-1}$ and red downflows at $-1 \mathrm{~km} \mathrm{~s}^{-1}$. Right: $x-z$ plane of the convection zone with the inserted flux rope.

(A color version of this figure is available in the online journal.)

Small-scale downflowing plasma merges and forms the largescale persistent downdrafts in the deep convection zone. The large-scale downdrafts are very important in the formation of pores during the building up of the active region, discussed in Section 3.1.

\subsection{Flux Rope}

The stratification of the atmosphere in the simulation domain is maintained self-consistently with the implementation of the thermodynamic processes in the model. The superadiabatic stratification provides an unstable background atmosphere for turbulent convective motion. As shown by Cheung et al. (2010), convective motion plays an important role in the formation of the sunspots and active region. However, the role of the turbulent convection in the emergence of the magnetic flux has not been clearly understood yet, particularly at larger scales. The aim of our simulation here is thus to study the transfer of the energy and magnetic flux of the rope during its rise from the deep convection zone, and its interaction with the surrounding turbulent medium. So after the generation of the convection zone with a hot corona, we linearly superimpose a flux rope upon the ambient magnetic field in the deep convection zone, at $z=-10 \mathrm{Mm}$, indicated by the dot-dashed line in the left panel of Figure 1. The initial flux rope is centrally buoyant and twisted along the $x$-axis, as described in Fan (2001) and Manchester et al. (2004) by the following equations:

$$
\mathbf{B}=\mathbf{B}_{\mathrm{amb}}+B_{0} e^{-r^{2} / a^{2}} \hat{\boldsymbol{x}}+q r B_{0} e^{-r^{2} / a^{2}} \hat{\boldsymbol{\theta}},
$$

$$
\begin{gathered}
\rho=\rho_{0}\left(1-\eta e^{-x^{2} / \lambda^{2}}\right), \\
\eta=\frac{\frac{1}{2}\left[B_{0} e^{-r^{2} / a^{2}}\right]^{2}\left[-1+\frac{1}{2} q^{2}\left(1-\frac{2 r^{2}}{a^{2}}\right)\right]}{p_{0}}, \\
p=p_{0}(1-\eta) .
\end{gathered}
$$

Here, $r$ is the distance from the axis of the rope and $a=1 \mathrm{Mm}$ is the radius of Gaussian decay. $q=-1.5$ is the twisting factor. $\lambda=6 \mathrm{Mm}$ is the length of the buoyant section. $p_{0}$ is the thermal pressure without the inserted flux rope. $B_{0}=50 \mathrm{kG}$ is the strength of the magnetic field at the axis of the rope, giving a minimum plasma beta value of $50 . \mathbf{B}_{\mathrm{amb}}$ is the ambient magnetic field. The total axial flux on the cross section of the initial flux rope is $1.5 \times 10^{21} \mathrm{Mx}$. Figure 2 shows the initial flux rope embedded with the convective plasma. The left 

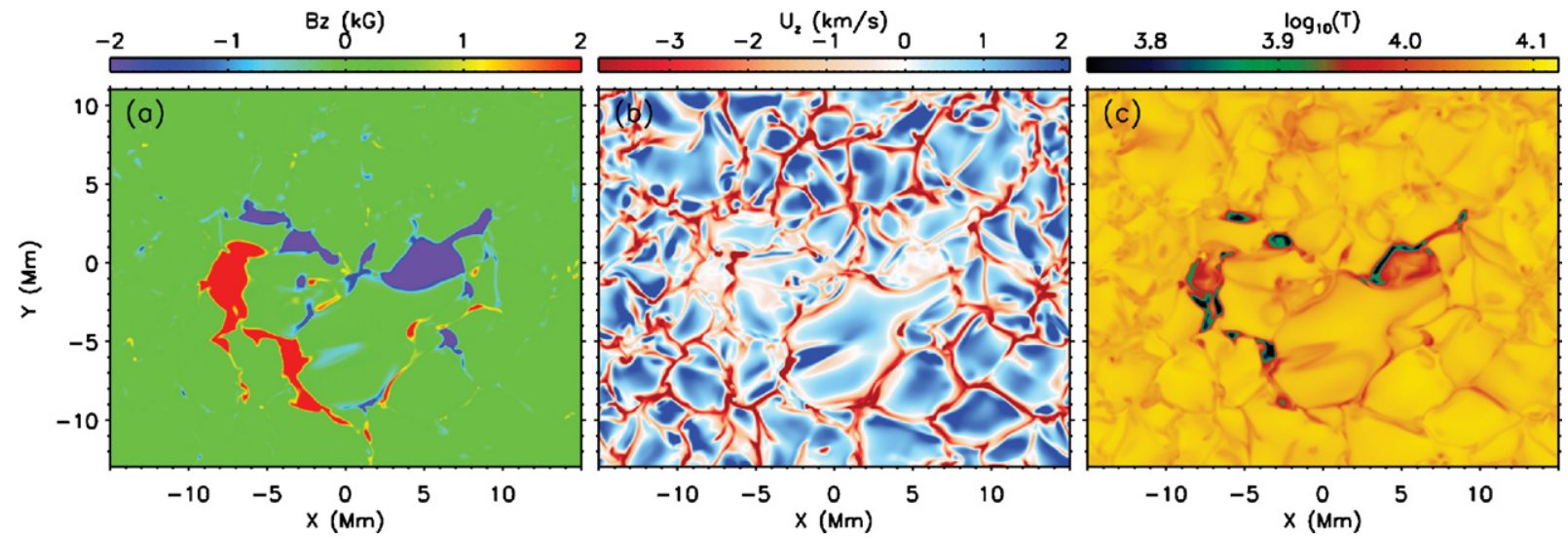

Figure 3. Structure of $B_{z}$ (a), $u_{z}$ (b), and $T$ (c) at $z=-1 \mathrm{Mm}$ plane at $t=5: 09: 00$.

(A color version of this figure is available in the online journal.)

panel illustrates three-dimensional isosurfaces of the large-scale convective flows around the flux rope in the deep convection zone, while the right panel shows the structure of $u_{z}$ in the convection zone on the $x-z$ plane with the flux rope. The complexity of the convective flows around the flux rope has a strong influence on the emerging process from the deep convection zone with large-scale downflows.

\section{RESULTS}

The flux rope is initially embedded in the deep convection zone, where it is buffeted by convective downflow and upflow. On the way from $z=-10 \mathrm{Mm}$ to the photosphere, the turbulent flows of various scales significantly reshape and redistribute the magnetic flux. On the photosphere, the magnetic flux first emerges as many small-area bipoles, which then sort themselves to form two large concentrations of opposite polarities via coalescence. Figure 3 shows the structure of the small active region at $z=-1 \mathrm{Mm}$. It contains two pores of opposite magnetic polarities, with smaller-size granules and cooler temperature. The formation of pores by the process of coalescence at the photosphere is revealed by both remote observations and previous numerical simulations (Vrabec 1974; Zwaan 1985; Cheung et al. 2010). However, the mechanism that transfers the magnetic flux from the tachocline, which is believed to be the origin of the active region magnetic field, to the solar surface is still to be determined. To date, global scale simulations of the solar interior cannot yet resolve individual flux tubes. Here, we examine the evolution of the magnetic flux rope in the deep convection zone and the photospheric and coronal response to its emergence.

\subsection{Formation of Pores}

The horizontal flux rope at $z=-10 \mathrm{Mm}$, shown by Figure 2, rises in the central section due to the depletion of the density and upwelling convective flows. However, the two ends of the central section are embedded in large-scale downflows present in the convection zone when the flux rope is initiated at $t=0: 00: 00$. The downflows are illustrated by the blue isosurfaces and color contours in Figure 2. Panel (a) of Figure 4 shows the structure of $u_{z}$ on the $x-z$ plane at $t=4: 50: 00$, when the downflows are still present in the convection zone at the two ends of the emerged flux rope. The region of the convective downflows in panel (a) of Figure 4 appears in great accordance with the black and green contour lines indicating the polarity of the pores. This consistency between the downflowing and magnetic concentrated regions suggests a causal relationship between the formation of the magnetic pores and the large-scale downflows. Figure 5 illustrates the temporal evolution of the three-dimensional magnetic field, colored by the $u_{z}$ value of the local plasma during the rising of the central section of the flux rope. The long-lasting, large-scale downflow, indicated by the blue color, drags down the two endpoints of the rising part and fixes them in the deep convection zone, forming an $\Omega$-shape emerged flux rope within $2.5 \mathrm{hr}$. The downflow maintains the pores and prevents the two pores of opposite polarities from separation or drifting apart, while the central section of the flux rope emerges and expands in the upper domain. Thus with the two footpoints deeply embedded and fixed in the convection zone, the emerged magnetic flux remains highly concentrated in a relatively small area at the photosphere.

In panel (b) of Figure 3 and panel (a) of Figure 4, small-scale convective granules in the near-surface layers appear inside each of the pores. Emonet \& Cattaneo (2001) also simulate the diminishing horizontal scale of the granules with increasing vertical magnetic field. While the magnetic field modifies the convective granules, the downward flows produce a bulb of colder plasma. The pressure imbalance with the surrounding material thus causes the flux tube to collapse and increases the strength of the magnetic field. The flux tube approaches equilibrium again with higher magnetic pressure balancing the surrounding material. With the convective collapse process (Parker 1978; Spruit 1979; Nagata et al. 2008), the magnetic field strength at the surface can be increased up to $4 \mathrm{kG}$, much higher than the equipartition field strength with the kinetic energy of the surrounding plasma. These near-surface processes, due to the convective motion, play a very important role in reorganizing the magnetic flux after its emergence at the photosphere. The magnetic field of the bipolar structures at the photosphere is intensified by the convective collapse in the near-surface layers, which is shown by panel (c) of Figure 4.

\subsection{Rotation of the Pores}

The magnetic flux rope travels through the $10 \mathrm{Mm}$ distance and approaches the photosphere after $2.5 \mathrm{hr}$. Figure 6 shows the evolution of the structure of the $B_{z}$ field at the photosphere, with arrows representing the horizontal velocity field. The magnetic field concentrates as narrow bands of bipolar fluxes, as shown in panels (a) and (b), in the regions between the major pores. The horizontal velocity, represented by the white arrows, reveals 

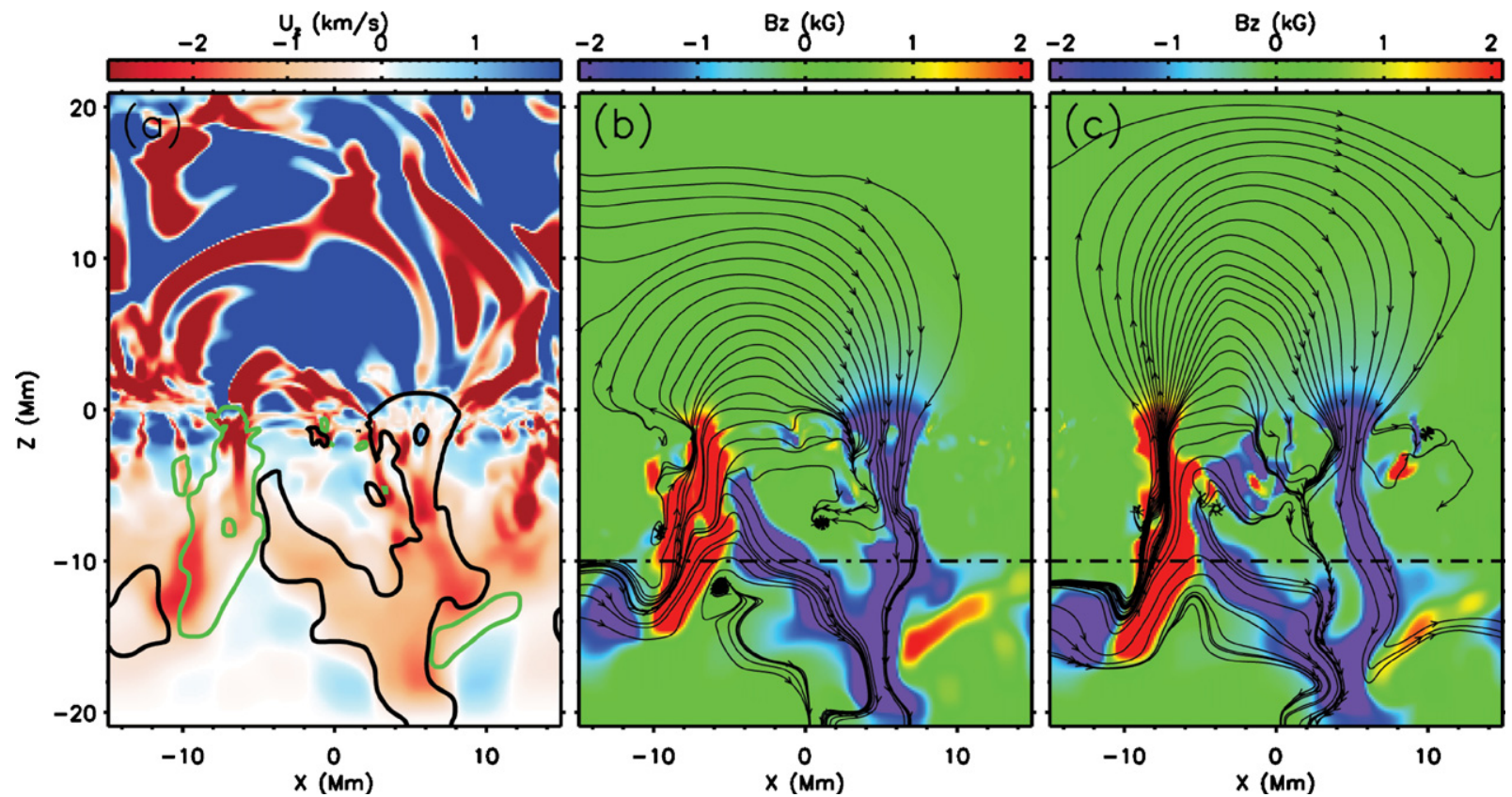

Figure 4. Structure of $u_{z}$ (a), $B_{z}$ (b), and $B_{z}$ (c) in the $y=0$ plane at $t=4: 50: 00$ ((a) and (b)) and $t=5: 22: 00$ (c). The black and green lines in panel (a) outline $B_{z}=$ $-1,1 \mathrm{kG}$, respectively. The dash-dotted line in panels (b) and (c) indicates the initial location of the axis of the inserted flux rope. And the black lines in panels (b) and (c) indicate the magnetic field lines by ignoring the $B_{y}$ component to show the direction of the field confined to the $x-z$ plane.
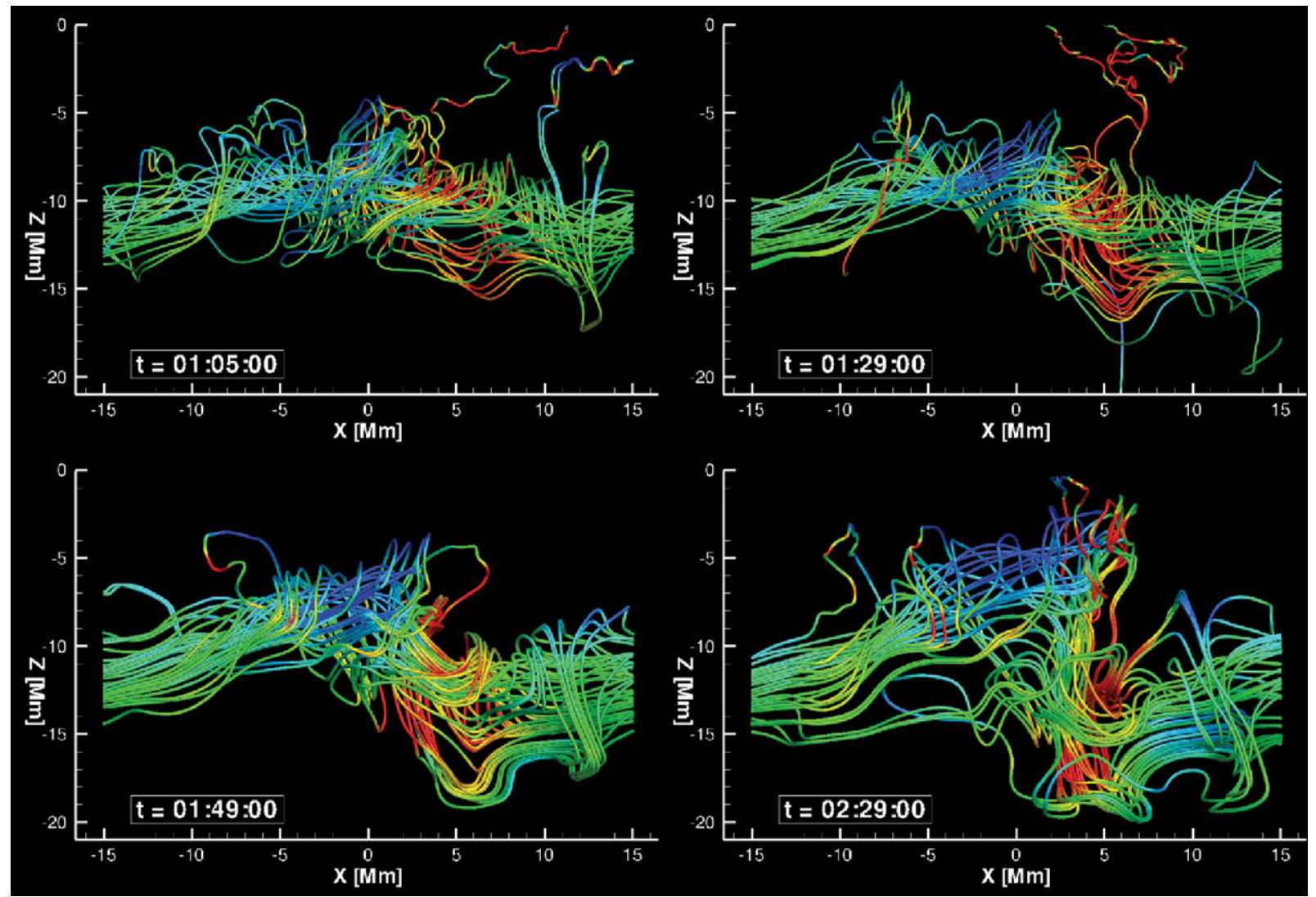

Figure 5. Temporal evolution of three-dimensional magnetic field lines colored by local $u_{z}$ values with red indicating downflows and blue upflows from -2 km $\mathrm{s}^{-1}$ to $1 \mathrm{~km} \mathrm{~s}^{-1}$, at $t=1: 05: 00,1: 29: 00,1: 49: 00$, and 2:29:00.

that the bipolar fluxes are moving in opposite directions, toward the major pores of the same polarity. For example, in panel (a), the rectangle outlines a region with flux emergence, where the negative flux is moving in the upper right direction into the negative pores and the positive flux bands are moving in the lower left direction into the positive pores. The coalescence of the small-scale fluxes into the major pores facilitates the accumulation of the magnetic flux on the surface and, therefore, 


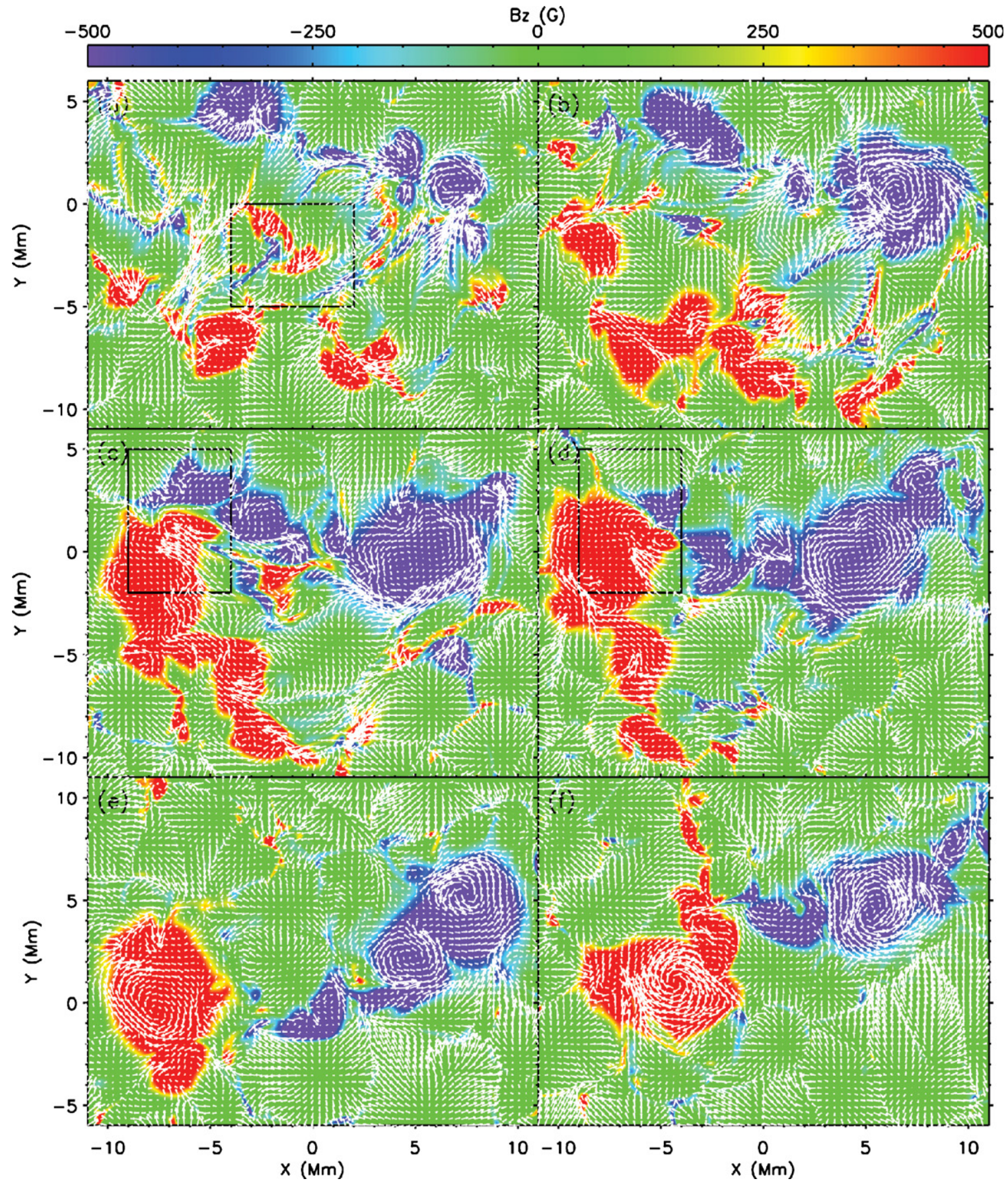

Figure 6. Structure of $B_{z}$ field at $z=0 \mathrm{Mm}$ at $t=3: 45: 00$ (a), 4:15:00 (b), 5:10:00 (c), 5:35:00 (d), 6:23:00 (e), and 7:41:00 (f). The arrows show the horizontal velocity field.

(An animation and a color version of this figure are available in the online journal.)

the formation of the large pores shown in panels (c) and (d). Cheung et al. (2010) simulate the formation of an active region and find that the counterstreaming motion of opposite polarities is driven by the Lorentz force.

The large pores of negative polarity show a coherent pattern of rotation at the photosphere after their formation in panel (a) of Figure 6. The rotation of the pores persists during the emergence and the increase of magnetic flux at the photosphere. However, the positive polarity on the left does not present a complete rotation pattern during the emerging phase, but the rotation is interrupted by the horizontal motions of the convective flow. The coherent rotation starts to develop on the positive pore after $5.5 \mathrm{hr}$, shown by panels (e) and (f) in Figure 6. Figure 7 illustrates the evolution of $B_{z}$ and the horizontal velocity fields at $z=-3 \mathrm{Mm}$, in the convection zone. Here, we observe a coherent rotation at this depth on the negative polarity as well. The question is then to what depth does the rotation extend. Thus we examine the structure of $u_{y}$ on the $y=0$ plane during the rising of the flux rope in the upper panels of Figure 8. The reversal of the direction of $u_{y}$ in the right side of the domain corresponds to the projection of the rotation of the negative polarity on the $y=0$ plane. The coherent rotation starts to extend downward at $t=4: 00: 00$ and approaches the depth of $-10 \mathrm{Mm}$ in 21 minutes (see panel (a)). Panel (c) shows 


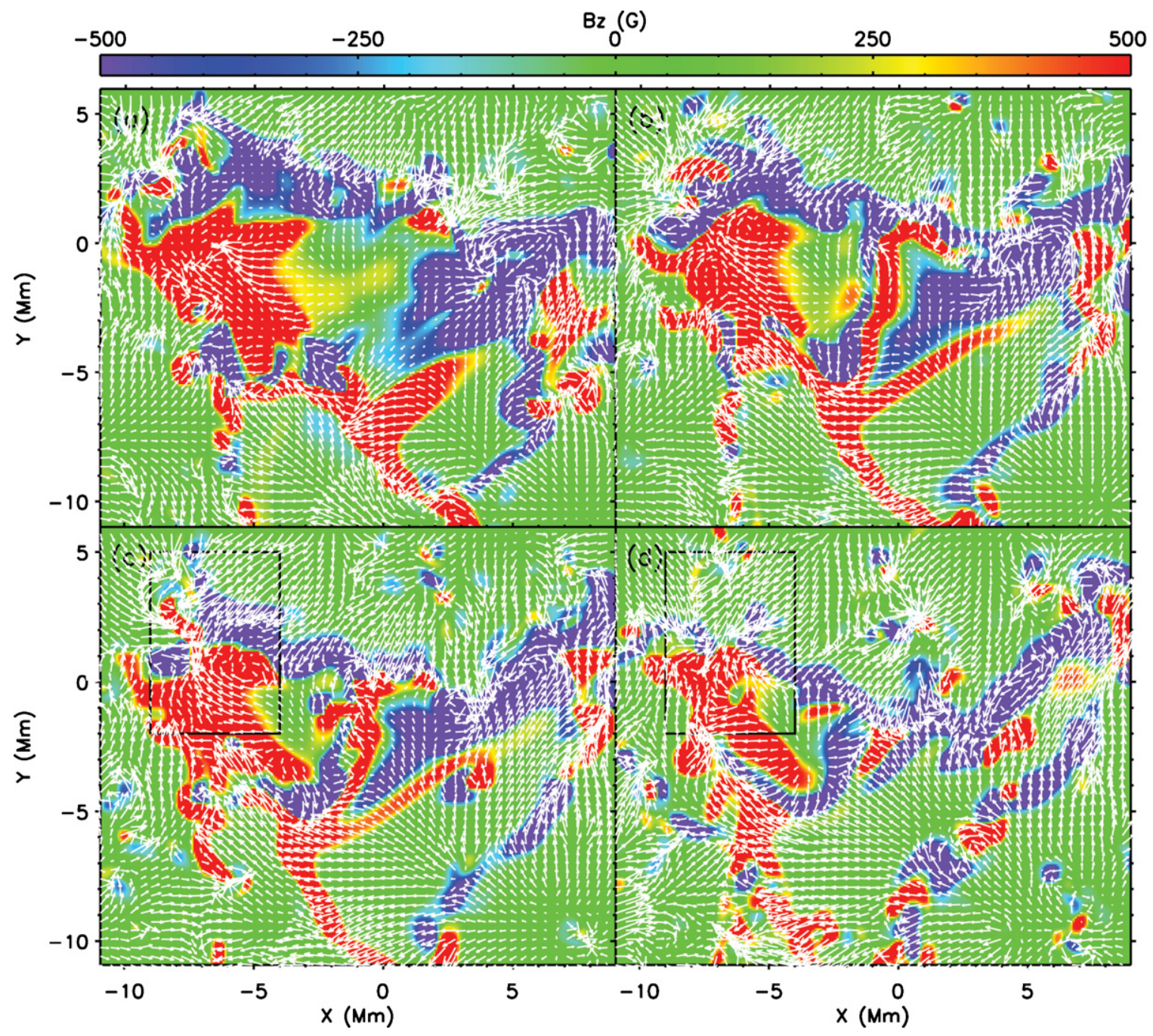

Figure 7. Structure of $B_{z}$ field at $z=-3 \mathrm{Mm}$ at $t=4: 40: 00$ (a), 5:00:00 (b), 5:10:00 (c), and 5:30:00 (d). The arrows show the horizontal velocity field.

(A color version of this figure is available in the online journal.)

a very coherent pattern of rotation on the negative polarity at $t=5: 13: 00$, while on the positive pore on the left, the rotation is not obvious.

Sunspot rotation has long been observed and studied in detail and has been found in association with CMEs (Brown et al. 2003; Kazachenko et al. 2009). The rotation mechanism for sunspots found at work in our simulation was first described in Parker (1979). Longcope \& Welsch (2000) simulate the increase of the helicity of coronal field due to the rotation of the photospheric footpoints in a dynamic model. Ideal MHD simulations by Fan (2009) illustrate the rotation driven by torsional Alfvén waves and the development of sigmoid-shaped field lines during the flux emergence from the top layer of the convection zone. Brown et al. (2003) and Min \& Chae (2009) study the behavior of the rotating sunspots in solar active regions and found that the rotation speed varies with time, radius, and angular spacing, with an increase in rotation speed in the penumbra. Kosovichev (1996) and Zhao \& Kosovichev (2003) find twists of the magnetic field and vortical flows around the sunspots in the convection zone underneath a rapidly rotating sunspot area. The flows underneath the negative pore in our simulation also exhibit a rotating pattern extending $10 \mathrm{Mm}$ down into the convection zone. The question is then what dynamic mechanism during flux emergence causes the observed variation in the rotation speed. Our simulation enables a detailed investigation of how sunspot rotation develops in the complex circumstances of the convection zone.

The development of the coherent rotation is accompanied by the presence of a strong Lorentz force in the azimuthal direction (see the upper panels in Figure 8), which is defined as $f_{y}=j_{z} B_{x}-j_{x} B_{z}$. The black and white contour lines in the upper panels of Figure 8 represent areas with strong negative and positive $f_{y}$, respectively. We find that the Lorentz force is driving the coherent rotation as a torsional Alfvén wave. At time $t=4: 21: 00$, shown by panel (a), the Lorentz force is in the same direction as $u_{y}$, thus acting to accelerate the rotation. However, the Lorentz force reverses direction at time $t=4: 37: 00$ (see panel (b)) and decelerates the rotation. Panel (c) reveals the structure of the Lorentz force at time $t=05: 13: 00$, which runs in the opposite direction of the rotation. The flux rope has rotated past its equilibrium point, and the Lorentz force reverses with the gradient of the azimuthal $\left(B_{y}\right)$ component of the field as the flux rope further emerges. The rotation of the pores untwists the field in the convection zone and produces a twisted magnetic field in the corona, as shown by the lower panels of Figure 8, which illustrates the $B_{y}$ field on the $y=0$ plane. As such, the rotation also provides a mechanism to build up the helicity and magnetic energy in the coronal 


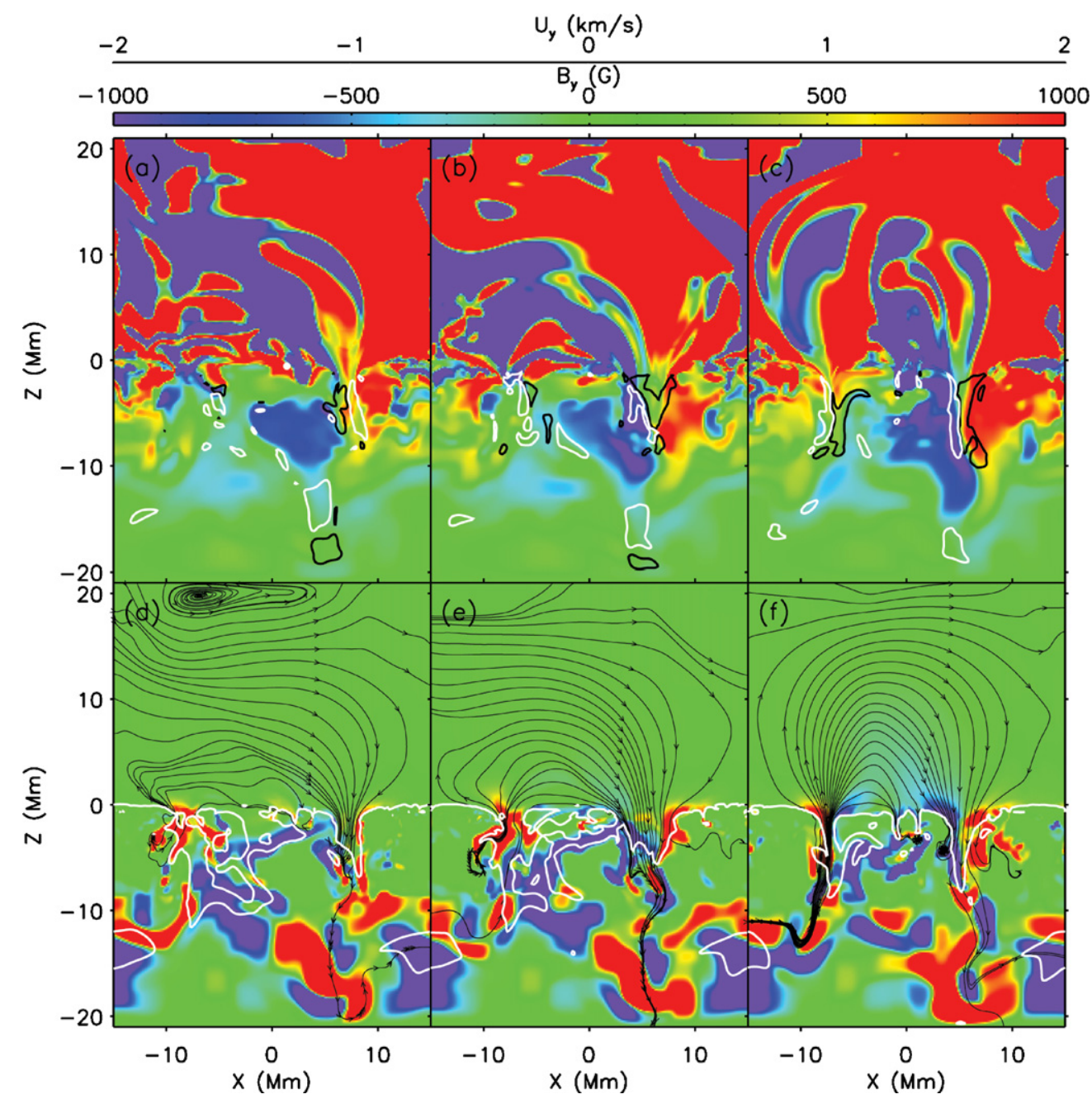

Figure 8. Structure of $u_{y}$ (upper panels) and $B_{y}$ (lower panels) at time $t=4: 21: 00$ ((a) and (d)), 4:37:00 ((b) and (e)), and 5:13:00 ((c) and (f)) in the $y=0$ plane The black and white contour lines in the upper panels represent regions with Lorentz force density $f_{y}=-20$ and 20 dyne $\mathrm{cm}^{3}$, respectively. And the black lines with arrows in the lower panels indicate streamlines of $\left(B_{x}, B_{z}\right)$ while white lines outline Alfvénic Mach number $u / u_{A}=1$.

(A color version of this figure is available in the online journal.)

magnetic field. The white lines in the lower panels outline where the Alfvénic Mach number $u / u_{A}=1$. The consistency between the structures of the Alfvénic Mach number and the Lorentz force $f_{y}$ suggests that in the pore region the magnetic field dominates over the plasma motion, and is responsible for the rotating flows.

In Figures 6 and 7, the positive polarity does not present as coherent a rotation as the negative polarity. However, in panels (c) and (d) of Figure 6, we observe a strong flux cancellation in the region outlined by the rectangles from time $t=5: 00: 00$ to $t=5: 35: 00$. Cheung et al. (2010) report that magnetic reconnection takes place within the U-loops formed by the convective downflows, which can remove the unsigned magnetic flux on the photosphere. In our simulation, the total amount of the magnetic flux that is canceled is $10 \%$ of the total unsigned flux on the photosphere. The flux cancellation strongly interrupts the coherency of the rotation on the positive polarity. The horizontal velocity fields exhibit a converging flow at the two opposite polarities of the canceled flux, both at the photosphere and in the convection zone, yielding a high gradient in the magnetic field. The close examination of the flux cancellation event will be the topic of a future paper. Another interesting feature we observe in our simulation is that the horizontal velocity field in Figure 7 shows a converging horizontal flow field around the magnetic flux region. This converging flow constrains the total area of the magnetic concentration, and thus prevents the magnetic pores from expansion and separation.

\subsection{Energy Fluxes}

The magnetic flux rope approaches the photosphere within $2.5 \mathrm{hr}$ after its initialization, and the photospheric magnetic flux, i.e., the emerged flux, reaches its maximum value at time $t=5: 11: 00$. Afterward, the magnetic pores start to decay slowly for the rest of the simulation. However, even at its maximum emergence, the magnetic flux rope is far from being fully above the photosphere. This is clearly shown by panels (b) and (c) of Figure 4, where the negative polarity is split into two parts, with one part emerging and forming pores while the other is sinking to the deep convection zone. The lower right panel of Figure 5 shows the three-dimensional magnetic field lines of the flux rope when it first approaches the photosphere. The red contour color over the field lines represents upflows while blue represents downflows. Only about half of the original flux rope is rising with upflows, while the other half remains almost stationary or sinks in the convection zone. 
It is of great interest to study the mechanism for transporting the initial magnetic flux from the deep convection zone into the photosphere, where the magnetic pores appear, and further into the coronal region. Here we calculate the temporal evolution of the total unsigned magnetic flux at the photosphere $(z=0)$, $z=-3 \mathrm{Mm}$ and $z=3 \mathrm{Mm}$, shown by the upper panel of Figure 10. At time $t=4: 49: 00$, the magnetic flux at $z=-3 \mathrm{Mm}$ reaches its maximum value of $2.63 \times 10^{21} \mathrm{Mx}$, which is most of the total unsigned flux $\left(3.04 \times 10^{21} \mathrm{Mx}\right)$ when the flux rope is initially bent.

At the photosphere, at $t=5: 11: 00$, the unsigned magnetic flux reaches its maximum of $1.37 \times 10^{21} \mathrm{Mx}, 45 \%$ of the total initial flux. The unsigned flux at $z=3 \mathrm{Mm}$ in the lower corona maximizes at $t=05: 29: 00$ with a value of $8.00 \times 10^{20} \mathrm{Mx}$. The ratio of the emerged unsigned flux with respect to the total initial flux decreases with increasing altitudes, from $86 \%$ at $z=-3 \mathrm{Mm}$ to $26 \%$ at $z=3 \mathrm{Mm}$. The sharp decrease from the convection zone to the photosphere is caused by the downflows in the near-surface layers that tend to return flux to the deep convection zone.

The question is then how the horizontal and vertical flows (driven both by convective motions and the Lorentz force) affect the emergence of magnetic flux and the transport of the magnetic energy. To address this question we next calculate the magnetic energy flux (Poynting flux) passing through three layers of the atmosphere: $z=-3,0$, and $3 \mathrm{Mm}$ using the following equations:

$$
\begin{gathered}
E_{\text {shear }}=-\int \frac{1}{4 \pi}\left(B_{x} u_{x}+B_{y} u_{y}\right) B_{z} d S \\
E_{\text {emerge }}=\int \frac{1}{4 \pi}\left(B_{x}^{2}+B_{y}^{2}\right) u_{z} d S
\end{gathered}
$$

The lower panel of Figure 10 illustrates the temporal evolution of the Poynting energy flux associated with the horizontal and vertical motions. We find that the energy flux associated with the vertical flows at $z=-3$ and $0 \mathrm{Mm}$ remains negative during the rising and decaying phase of the flux emergence. There are several transient positive pulses of energy fluxes by the vertical motions at $z=-3 \mathrm{Mm}$, at times $t=2: 09: 00$ and $t=2: 57: 00$. These transient positive pulses represent the energy transport when the magnetic flux first emerges to the surface with upflows, as shown by the lower right panel of Figure 5. Each of these pulses is followed by a sharp increase in the energy flux associated with the horizontal flows and a reversal of the energy flux by vertical flows. This time evolution of energy shows a process of magnetic flux emergence at the near-surface layers with convective flows: magnetic flux emerges at the surface as bipoles with upflowing motion, then they are quickly pulled apart by the horizontal flows, and concentrate in the downdrafts. This process then leads to a positive energy flux by the upflows, followed by an increasing energy flux by the horizontal motions, and a negative energy flux associated with the downdrafts.

Panels (a) and (b) in Figure 9 show the $B_{z}$ field at $z=-3$ and $0 \mathrm{Mm}$, overlaid by white lines showing downdrafts. The concentration of the magnetic flux in the downdrafts explains the negative energy flux associated with the vertical flows in the lower panel from $3.0 \mathrm{hr}$ to $5.0 \mathrm{hr}$ while the total magnetic flux is still increasing in the upper panel. During this time period, more than $60 \%$ of the total unsigned flux is in the downflowing region for both $z=-3$ and $0 \mathrm{Mm}$ layers. At time $t=5: 11: 00$, when the photospheric unsigned flux maximizes, $70 \%$ of emerged flux is concentrated in the downdrafts. Panels (c) and (d) show the Poynting fluxes at the photosphere associated with the vertical and horizontal flows, defined as

$$
\begin{gathered}
F_{\text {shear }}=-\frac{1}{4 \pi}\left(B_{x} u_{x}+B_{y} u_{y}\right) B_{z} \\
F_{\text {emerge }}=\frac{1}{4 \pi}\left(B_{x}^{2}+B_{y}^{2}\right) u_{z}
\end{gathered}
$$

$F_{\text {emerge }}$ is negative in the magnetic polarities while positive in the areas between the polarities, where the magnetic flux is emerging. In the pores, the energy flow is instead dominated by the $F_{\text {shear }}$, shown by panel (d). At $z=3 \mathrm{Mm}$, in the corona, the energy flux by the vertical flows shows short periods of positive values. The increased energy flux is driven upward by convectively driven magnetoacoustic shocks and could be interpreted in terms of the dynamics of Type I spicules (see, e.g., Hansteen et al. 2006; Martínez-Sykora et al. 2009).

The Poynting flux associated with the horizontal flows dominates the energy transport during the flux emergence. At the photosphere, the total energy transport by this flux is $1.35 \times 10^{32} \mathrm{erg}$, while vertical flows transport $5.77 \times 10^{31} \mathrm{erg}, 42 \%$ of the energy back to the convection zone by the end of the simulation. The horizontal flows here include the rotation of the magnetic pores, the separating motion of the small bipoles, and the shearing motion along PILs. The rotation of the pores is discussed in Section 3.2, which transports both the magnetic energy and helicity into the corona region. The extension of the rotation motion into the deep convection zone shown by Figure 8 greatly impacts the spatial distribution of the Poynting flux by the rotation. Figure 6 shows the separating process of the small bipoles, which tend to move apart from each other after their emergence at the photosphere and merge into large pores with the same polarity via this self-sorting process. The horizontal separating flow on the small bipoles builds up energy in the near-surface region. In panels (c) and (d) of Figures 6 and 7 , the black rectangles outline the area with a large-scale magnetic flux cancellation. The converging flows across the PIL build up the magnetic gradient in this area, and the shearing flow along the PIL, shown in panels (c) and (d) of Figure 6, drives the magnetic field lines along the PIL and builds up a highly sheared magnetic field configuration, which plays an important role in eruptive events such as flares, filament eruptions, and CMEs (Mikic \& Linker 1994; Wu \& Guo 1997; Antiochos et al. 1999). A detailed analysis of this flux cancellation event will be the topic of a future paper.

\section{DISCUSSION AND CONCLUSIONS}

In Sections 3.1 and 3.2, we examined the structures of the vertical and horizontal velocity fields separately and their influence on the emerging magnetic flux rope. The persistent large-scale downflows help form and maintain the bipolar pores of uniform polarity in the deep convection zone, while the smallscale downflows in the near-surface layers intensify the strength of the emerged flux tube by convective collapse, as suggested by Parker (1978) and found in previous simulations by Stein $\&$ Nordlund (2006). At the photosphere, the horizontal flows act on the small newly emerged bipoles to not only separate opposite polarities but also sort them into large pores of uniform polarity. This coalescence of small-scale fluxes enables the formation of the magnetic pores with an unsigned flux of up to $1.37 \times 10^{21} \mathrm{Mx}$, 


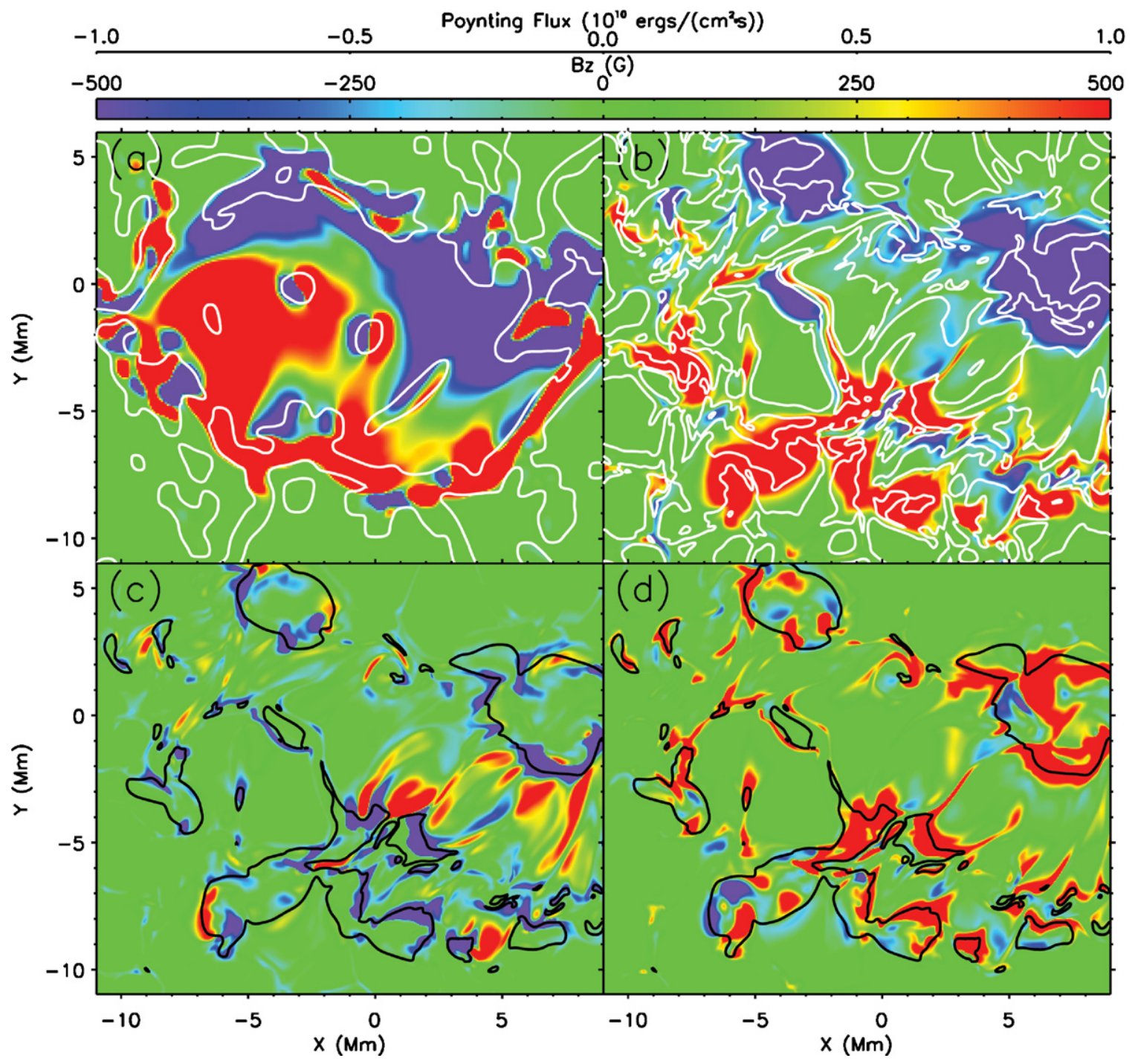

Figure 9. Structure of $B_{z}$ field at $z=-3 \mathrm{Mm}$ at $t=3: 40: 00$ (a), $B_{z}$ field at $z=0 \mathrm{Mm}$ at $t=4: 03: 00$ (b) and Poynting fluxes associated with vertical (c) and horizontal flows (d) at $z=0 \mathrm{Mm}$ at $t=4: 03: 00$. The white lines in panels (a) and (b) indicate downflowing regions with $u_{z}=-0.1 \mathrm{~km} \mathrm{~s}^{-1}$, and the black lines in panels (c) and (d) show regions with $\left|B_{z}\right|=500 \mathrm{G}$.

(A color version of this figure is available in the online journal.)

which is limited by the scale of our simulation. The horizontal flows of the magnetic pores exhibit a coherent rotation extending down to the deep convection zone, which is clearly driven by the Lorentz force as was found in earlier simulations (Longcope \& Welsch 2000; Fan 2009). Our simulation illustrates that this rotation mechanism operates in a realistic convection zone but requires the coalescence of a well-formed pore and that complex interactions that break the symmetry of the pore (such as flux cancellation) may disrupt the rotation.

The energy transport due to the horizontal and vertical flows is calculated in the domain from the convection zone into the corona. Abbett \& Fisher (2011) find the Poynting energy flux flows into the interior below the visible surface and flows into the corona above it and suggest surface convection as the energy source of the separatrix in the energy flux. Our study shows a negative total energy flux at $z=-3 \mathrm{Mm}$, and positive at $z=$ 0 and $3 \mathrm{Mm}$, which agrees with this result. The energy flux is initially dominated by the emerging flow on the magnetic flux, quickly after which opposite polarities separate, giving rise to an increase in the energy flux associated with the horizontal flows. This general trend has been found in early work, e.g., Magara \& Longcope (2003), Manchester et al. (2004), and Fang et al. (2010), but these works did not illustrate the extent to which downflows control energy transport in the convection zone. In the convection zone and at the photosphere, the flux concentrates in the downflow drafts, yielding a negative energy flux associated with the vertical motion. The horizontal flows are thus the dominating carriers of the energy transport into the corona. We identify three types of horizontal flows in Section 3.2: the rotation, the separating motion of the bipoles, and the shearing motion along PILs. The rotation and shearing both transfer the magnetic energy and helicity into the corona. And it is the separating and shearing motions that build up the magnetic energy in the near-surface region, which is crucial for the eruptive events.

In our simulation, we also find an area with cancellation of magnetic flux up to $10^{20} \mathrm{Mx}$ within $0.5 \mathrm{hr}$, shown by panels (c) and (d) of Figure 6. The coronal response to this large-scale flux cancellation is of great interest. Green et al. (2011) find the evolution of coronal fields into a highly sheared arcade and 


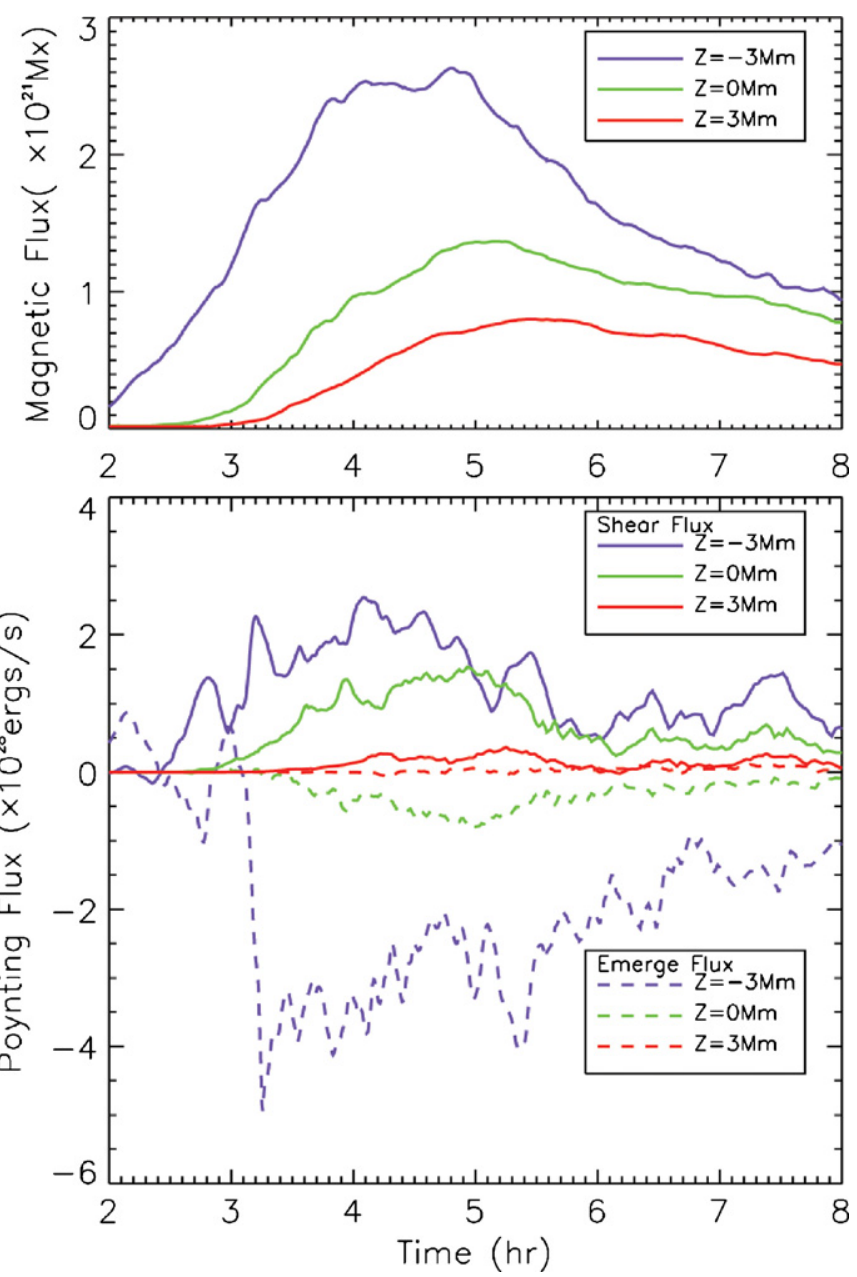

Figure 10. Temporal evolution of magnetic flux (upper) and energy flux (lower) associated with the horizontal flows (solid) and vertical flow (dashed) at $z=$ -3 (purple), 0 (green), and 3 (red) Mm from time $t=2: 00: 00$ to $t=8: 00: 00$.

then a sigmoid in an active region where one-third of the flux is canceled. Whether the canceled flux in our simulation produces a flux rope in the coronal region will be the topic of a future paper. Another interesting feature is the decay of the magnetic pores from time $t=6: 00: 00$ to $t=8: 00: 00$, shown by Figure 10. van Ballegooijen \& Mackay (2007) suggest that the submerged magnetic field repairs the toroidal flux ropes from which the initial flux emerged. The decayed magnetic flux thus may play a fundamental role in replacing the magnetic field in the solar interior.

This work was supported by NASA grants NNG06GD62G, NNX07AC16G and NSF grants ATM 0642309 and AGS 1023735. W.M. IV was also funded by NASA grant LWS NNX09AJ78G. W.P.A. was funded in part by NSF grants ATM-0641303 and AGS-1048318, and through the NASA Living with a Star TR\&T program (NNX08AQ30G) and the NASA Heliophysics Theory Program (NNX08AI56G and NNX11AJ65G). The simulations described here were carried out on the Pleiades system at the NASA Advanced Supercomputing (NAS) Facility and Bluefire cluster at NCAR.

\section{REFERENCES}

Abbett, W. P. 2007, ApJ, 665, 1469

Abbett, W. P., \& Fisher, G. H. 2003, ApJ, 582, 475

Abbett, W. P., \& Fisher, G. H. 2011, Sol. Phys., doi:10.1007/s11207-011-9817-3

Antiochos, S. K., DeVore, C. R., \& Klimchuk, J. A. 1999, ApJ, 510, 485

Archontis, V., \& Török, T. 2008, A\&A, 492, L35

Brown, D. S., Nightingale, R. W., Alexander, D., et al. 2003, Sol. Phys., 216, 79

Canfield, R. C., Hudson, H. S., \& McKenzie, D. E. 1999, Geophys. Res. Lett., 26, 627

Cheung, M. C. M., Rempel, M., Title, A. M., \& Schüssler, M. 2010, ApJ, 720, 233

Cheung, M. C. M., Schüssler, M., \& Moreno-Insertis, F. 2007, A\&A, 467, 703

Emonet, T., \& Cattaneo, F. 2001, ApJ, 560, L197

Fan, Y. 2001, ApJ, 554, L111

Fan, Y. 2004, Living Rev. Sol. Phys., 1, 1

Fan, Y. 2008, ApJ, 676, 680

Fan, Y. 2009, ApJ, 697, 1529

Fang, F., Manchester, W., Abbett, W. P., \& van der Holst, B. 2010, ApJ, 714, 1649

Green, L. M., Kliem, B., \& Wallace, A. J. 2011, A\&A, 526, A2

Gudiksen, B. V., Carlsson, M., Hansteen, V. H., et al. 2011, A\&A, 531, A154

Hagenaar, H. J., Schrijver, C. J., \& Title, A. M. 2003, ApJ, 584, 1107

Hansteen, V. H., De Pontieu, B., Rouppe van der Voort, L., van Noort, M., \& Carlsson, M. 2006, ApJ, 647, L73

Jouve, L., \& Brun, A. S. 2009, ApJ, 701, 1300

Kazachenko, M. D., Canfield, R. C., Longcope, D. W., et al. 2009, ApJ, 704, 1146

Kosovichev, A. G. 1996, ApJ, 461, L55

Longcope, D. W., \& Welsch, B. T. 2000, ApJ, 545, 1089

MacTaggart, D., \& Hood, A. W. 2009, A\&A, 507, 995

Magara, T., \& Longcope, D. W. 2003, ApJ, 586, 630

Manchester, W. 2003, J. Geophys. Res. (Space Phys.), 108, 1162

Manchester, W., IV. 2007, ApJ, 666, 532

Manchester, W. 2008, in ASP Conf. Ser. 383, Subsurface and Atmospheric Influences on Solar Activity, ed. R. Howe, R. W. Komm, K. S. Balasubramaniam, \& G. J. D. Petrie (San Francisco, CA: ASP), 91

Manchester, W., IV, Gombosi, T., DeZeeuw, D., \& Fan, Y. 2004, ApJ, 610, 588

Manchester, W., \& Low, B. C. 2000, Phys. Plasmas, 7, 1263

Martínez-Sykora, J., Hansteen, V., \& Carlsson, M. 2008, ApJ, 679, 871

Martínez-Sykora, J., Hansteen, V., DePontieu, B., \& Carlsson, M. 2009, ApJ, 701,1569

Mikic, Z., \& Linker, J. A. 1994, ApJ, 430, 898

Min, S., \& Chae, J. 2009, Sol. Phys., 258, 203

Nagata, S., Tsuneta, S., Suematsu, Y., et al. 2008, ApJ, 677, L145

Nordlund, Å., Stein, R. F., \& Asplund, M. 2009, Living Rev. Sol. Phys., 6, 2

Parker, E. N. 1978, ApJ, 221, 368

Parker, E. N. (ed.) 1979, Cosmical Magnetic Fields: Their Origin and Their Activity (New York: Oxford University Press)

Parnell, C. E., DeForest, C. E., Hagenaar, H. J., et al. 2009, ApJ, 698, 75

Pevtsov, A. A., Fisher, G. H., Acton, L. W., et al. 2003, ApJ, 598, 1387

Powell, K. G., Roe, P. L., Linde, T. J., Gombosi, T. I., \& de Zeeuw, D. L. 1999, J. Comput. Phys., 154, 284

Rempel, M. 2011, ApJ, 729, 5

Rempel, M., Schüssler, M., \& Knölker, M. 2009, ApJ, 691, 640

Rogers, F. J. 2000, Phys. Plasmas, 7, 51

Shibata, K., Tajima, T., Steinolfson, R. S., \& Matsumoto, R. 1989, ApJ, 345, 584

Spruit, H. C. 1979, Sol. Phys., 61, 363

Stein, R. F., Lagerfjärd, A., Nordlund, Å., \& Georgobiani, D. 2011, Sol. Phys., 268,271

Stein, R. F., \& Nordlund, Å. 2006, ApJ, 642, 1246

Tortosa-Andreu, A., \& Moreno-Insertis, F. 2009, A\&A, 507, 949

van Ballegooijen, A. A., \& Mackay, D. H. 2007, ApJ, 659, 1713

Vögler, A., Shelyag, S., Schüssler, M., et al. 2005, A\&A, 429, 335

Vrabec, D. 1974, in IAU Symp. 56, Chromospheric Fine Structure, ed. R. G. Athay (Dordrecht: Reidel), 201

Wu, S. T., \& Guo, W. P. 1997, Adv. Space Res., 20, 2313

Zhao, J., \& Kosovichev, A. G. 2003, ApJ, 591, 446

Zwaan, C. 1985, Sol. Phys., 100, 397 